\title{
Surface Hardness of Resin Cement Polymerized under Different Ceramic Materials
}

\author{
Pimmada Kesrak $^{1}$ and Chalermpol Leevailoj ${ }^{2}$ \\ ${ }^{1}$ Department of Conservative Dentistry, Faculty of Dentistry, Prince of Songkla University, Hatyai, Songkhla 90112, Thailand \\ ${ }^{2}$ Esthetic Restorative and Implant Dentistry Program, Faculty of Dentistry, Chulalongkorn University, Henri-Dunant Road, \\ Patumwan, Bangkok 10330, Thailand \\ Correspondence should be addressed to Pimmada Kesrak, kpimmada@gmail.com
}

Received 3 November 2011; Revised 26 December 2011; Accepted 17 January 2012

Academic Editor: J. Anthony Von Fraunhofer

Copyright (๑) 2012 P. Kesrak and C. Leevailoj. This is an open access article distributed under the Creative Commons Attribution License, which permits unrestricted use, distribution, and reproduction in any medium, provided the original work is properly cited.

Objectives. To evaluate the surface hardness of two light-cured resin cements polymerized under different ceramic discs. Methods. 40 experimental groups of 2 light-cured resin cement specimens (Variolink Veneer and NX3) were prepared and polymerized under 5 different ceramic discs (IPS e.max Press HT, LT, MO, HO, and Cercon) of 4 thicknesses $(0.5,1.0,1.5$, and 2.0 mm), Those directly activated of both resin cements were used as control. After light activation and $37^{\circ} \mathrm{C}$ storage in an incubator, Knoop hardness measurements were obtained at the bottom. The data were analyzed with three-way ANOVA, $t$-test, and oneway ANOVA. Results. The KHN of NX3 was of significantly higher than that of Variolink Veneer $(P<0.05)$. The KHN of resin cement polymerized under different ceramic types and thicknesses was significant difference $(P<0.05)$. Conclusion. Resin cements polymerized under different ceramic materials and thicknesses showed statistically significant differences in KHN.

\section{Introduction}

Currently, there is an increased demand for esthetic restorations, especially all-ceramic restorations, including all-ceramic crowns, inlays, onlays, and veneers. Therefore, many new ceramic systems have been developed. Current ceramic materials have numerous superior properties, such as their esthetic lifelike appearance, biocompatibility, chemical stability, and high compressive strength. Continuing development in ceramic core materials has made all-ceramic restorations a more valuable clinical option. Ceramic core materials are categorized into 3 different types: glass, alumina based, and zirconia [1].

In addition to the physical properties of ceramics, luting materials are important for the longevity of ceramic restorations. The use of resin cement in combination with a dental adhesive will strengthen all-ceramic restorations and influence the longevity of the restoration [2]. Furthermore, resin cement has an effect on the esthetics of restorations due to the color of the cement. Because ceramic is a translucent material, using tooth-colored resin cement under ceramic restorations allows the observation of the color of the cement and improves the esthetics of the restoration. Alternatively, some resin cements can obscure dark-colored teeth and that may affect the color of restoration.

Resin cements are composed of methacrylate or BisGMA, similar to resin composite. According to the activation mode, resin cements are usually divided into three groups: chemically activated (self-cured), photoactivated (light-cured), and dual-cured, in which the polymerization was affected by both chemical and light activation [3]. Light-cured resin cements use a photoinitiator, primarily camphorquinone; the polymerization process begins when activated by light from the light-curing unit. Light-cured resin cements are often preferred to chemical-cured and dual-cured resin cements because of their on-demand polymerization characteristic. Moreover, this type of resin cement allows for easier manipulation due to the lack of preparation process, such as mixing before use, which results in decreased air incorporation into the cement and also decreased color instability. However, light-cured resin cements also have a limitation associated with the polymerization process as they 
require sufficient light to initiate and maintain polymerization, especially in deep cavities or thick restorations that may attenuate the light from the light-curing unit. Incomplete polymerization of materials will affect both physical and biological properties, such as surface hardness, color instability, toxicity from residual monomer [4-7], and decreased bond strength between tooth and restoration (which can also decrease the longevity of the restoration).

The effectiveness of light to initiate polymerization of resin-based materials requires the appropriate wavelength determined by the type of photoinitiator incorporated in the resin-based material [8]. Camphorquinone is effectively activated by light at a wavelength range of $375-500 \mathrm{~nm}$, with a peak maximum absorption at $468-470 \mathrm{~nm}[9,10]$ and also with a light intensity high enough to activate polymerization. Factors affecting light efficiency include the light-curing unit, exposure time, and any object between the light tip and the resin cement [11-13]. Recently, the light emitting diode (LED) light-curing units have become very popular among dental practitioners because of advantages over existing light-curing units. The LED produces a narrow spectrum of light that falls within the absorption spectrum of the camphorquinone [14]. Furthermore, an LED has a lower power requirement and is powered by a rechargeable battery which makes the device a cordless, portable, and lightweight unit with a longer lifespan.

Surface hardness testing is one aspect of the polymerization measurement method [15-17]. It provides a strong correlation to the light intensity used in polymerization activation [18-20]. Hence, this study aimed to evaluate the surface hardness of two light-cured resin cements polymerized under different types and thicknesses of ceramic discs.

\section{Materials and Methods}

For IPS e.max Press (Ivoclar Vivadent, Liechtenstein) ceramic groups, the ceramic discs were fabricated from IPS e.max Press high-translucency ingot (HT-A1), low-translucency ingot (LT-A1), medium-opacity ingot (MO-0), and high-opacity ingot $(\mathrm{HO}-0)$, with a $10 \mathrm{~mm}$ diameter and thicknesses of $0.5,1.0,1.5$, and $2.0 \mathrm{~mm}$. The wax patterns, $10 \mathrm{~mm}$ in diameter with a level of thickness exceeding $0.2 \mathrm{~mm}$ each, were fabricated to the ceramic discs by the lost wax, heat press process. The ceramic discs were polished with an automatic polishing machine (DPS 3200, IMPTECH, South Africa) and silicon carbide paper nos. 400, 600, 800, and 1200, respectively. The final thickness was measured by a digital micrometer (Mitutoyo, Japan).

For Cercon (DeguDent, Germany) ceramic groups, the ceramic discs were fabricated from a Cercon base (white) to a $10 \mathrm{~mm}$ diameter and thicknesses of $0.5,1.0,1.5$, and $2.0 \mathrm{~mm}$. The Cercon base was cut into the framework in a circular shape and trimmed by the silicon carbide paper. The framework required a $30 \%$ greater diameter and thickness to compensate for shrinkage during the sintering process. After sintering, the ceramic discs were polished and measured, as previously described.
The resin cements used in this study were Variolink Veneer (Ivoclar Vivadent, Liechtenstein) shade high value +3 and NX3 Nexus Third Generation (Kerr Corporation, USA) shade white opaque. The resin cement was inserted into a black PVC mold with a centered hole $6.0 \mathrm{~mm}$ in diameter and $0.5 \mathrm{~mm}$ deep. A glass slab $(0.04 \mathrm{~mm}$ thick) was placed above the mold and resin cement. The resin cement underwent light activation in two modes: direct light activation (control) or light activation through ceramic discs (experimental), with 5 ceramic groups and 4 thicknesses each. In the experimental groups, the ceramic discs were placed between the tip of the light guide of the light-curing unit and the glass slab covering the resin cement before activation. The resin cement was light activated by an LED light-curing unit (Demi, Kerr Corporation, USA) with an irradiance of $1,450 \mathrm{~mW} / \mathrm{cm}^{2}$ for 40 seconds, in contact with the ceramic material or glass slab. The intensity of the light-curing units was measured with a hand-held radiometer (L.E.D. radiometer by Demitron, Kerr Corporation, USA) that was recalibrated after 10 times of usage. The specimens were stored in an incubator (CONTHERM $160 \mathrm{M}$, CONTHERM Scientific Ltd., New Zealand) at $37^{\circ} \mathrm{C}$ for approximately 24 hours. Forty two groups of 12 resin cement specimens each were tested.

The micro-hardness tester (FM-700e TYPE D, FUTURETECH, Japan) with 50-gram force for 15 seconds was used for Knoop hardness testing. Three indentations were made on the bottom surface of each specimen, with a $1 \mathrm{~mm}$ distance between indentations, and the means were then calculated. Measurements were made under 40x magnification.

Statistical Analysis. SPSS software version 17 was used to analyze the results at a 0.05 significance level $(P<0.05)$. The effects of resin cement types, ceramic types, and thicknesses of ceramic on resin cement hardness were analyzed using three-way ANOVA. The independent $t$-test was used to compare the 2 different types of resin cement. To compare the surface hardness of light-curing resin cement cured through different thicknesses of ceramics, we used one-way ANOVA and Tukey's multiple comparison test. Finally, the one-way ANOVA and Tukey's multiple comparison test were used to compare the differences of the resin cement surface hardness cured through different types of ceramic having the same thickness.

\section{Results}

Table 1 shows the surface hardness value of two resin cements when polymerized under different types and thicknesses of ceramic discs. The surface hardness of Variolink Veneer was statistically lower than that of NX3 in all experimental groups. The surface hardness of both resin cements (Variolink Veneer and NX3) when polymerized under ceramic discs presented statistically significant differences from the control. For Variolink Veneer, the surface hardness of resin cement polymerized under IPS e.max Press HT $2.0 \mathrm{~mm}$, LT $2.0 \mathrm{~mm}, \mathrm{MO} 1.5,2.0 \mathrm{~mm}$, HO $1.0,1.5$, and, $2.0 \mathrm{~mm}$ and Cercon $1.0,1.5$, and $2.0 \mathrm{~mm}$ was significantly lower than that 
TABLE 1: The mean $(\mathrm{KHN}) \pm$ standard deviation of surface hardness of two resin cements when polymerized under different ceramic a.

\begin{tabular}{|c|c|c|}
\hline \multirow{2}{*}{$\begin{array}{l}\text { Ceramic/thickness } \\
(\mathrm{mm})\end{array}$} & \multicolumn{2}{|c|}{$\mathrm{KHN}($ mean $\pm \mathrm{SD})$} \\
\hline & Variolink Veneer & NX3 \\
\hline Control & $21.64 \pm 3.7^{1}$ & $30.28 \pm 1.8^{2}$ \\
\hline e.max HT/0.5 & $19.39 \pm 3.8^{1 \mathrm{Aa}}$ & $30.16 \pm 1.8^{2 \mathrm{Ne}}$ \\
\hline e.max HT/1.0 & $18.39 \pm 3.3^{1 \mathrm{ABb}}$ & $29.80 \pm 2.2^{2 \mathrm{Nf}}$ \\
\hline e.max HT/1.5 & $17.16 \pm 2.9^{1 \mathrm{ABC}}$ & $27.79 \pm 1.9^{2 \mathrm{Nh}}$ \\
\hline e.max HT/2.0 & $15.38 \pm 2.1^{1 \mathrm{Bd}^{*}}$ & $24.83 \pm 3.3^{2 \mathrm{Ok}^{*}}$ \\
\hline e.max LT/0.5 & $19.42 \pm 3.2^{1 \mathrm{Ca}}$ & $28.30 \pm 2.3^{2 \mathrm{Pe}}$ \\
\hline e.max LT/1.0 & $18.40 \pm 3.1^{1 \mathrm{CDb}}$ & $27.29 \pm 2.3^{2 \mathrm{POfg}}$ \\
\hline e.max LT/1.5 & $16.45 \pm 3.2^{1 \mathrm{CDc}}$ & $26.90 \pm 2.1^{2 \mathrm{QRhi}^{*}}$ \\
\hline e.max LT/2.0 & $15.30 \pm 3.2^{1 \mathrm{Dd}^{*}}$ & $22.99 \pm 2.1^{2 \mathrm{Rk}^{*}}$ \\
\hline e.max $\mathrm{MO} / 0.5$ & $19.43 \pm 3.2^{1 \mathrm{Ea}}$ & $28.46 \pm 1.5^{2 \mathrm{Se}}$ \\
\hline e.max $\mathrm{MO} / 1.0$ & $17.97 \pm 3.2^{1 \mathrm{EFb}}$ & $27.45 \pm 2.2^{2 \mathrm{Sfg}}$ \\
\hline e.max $\mathrm{MO} / 1.5$ & $16.47 \pm 3.3^{1 \mathrm{EFc}}$ & $26.31 \pm 1.8^{2 \mathrm{Shi}^{*}}$ \\
\hline e.max $\mathrm{MO} / 2.0$ & $14.81 \pm 2.8^{1 \mathrm{Fd}^{*}}$ & $23.81 \pm 2.4^{2 \mathrm{Tkm} *}$ \\
\hline e.max HO/0.5 & $19.81 \pm 3.9^{1 \mathrm{Ga}}$ & $29.48 \pm 2.6^{2 \mathrm{Ue}}$ \\
\hline e.max $\mathrm{HO} / 1.0$ & $18.15 \pm 3.4^{1 \mathrm{GHb}}$ & $27.16 \pm 2.4^{2 \mathrm{UVfg}} *$ \\
\hline e.max $\mathrm{HO} / 1.5$ & $16.34 \pm 2.8^{1 \mathrm{HJc} *}$ & $25.51 \pm 2.3^{2 \mathrm{Vhi}^{*}}$ \\
\hline e.max $\mathrm{HO} / 2.0$ & $13.01 \pm 2.1^{1 \mathrm{Jd}^{*}}$ & $20.08 \pm 2.6^{2 \mathrm{Wkm} *}$ \\
\hline Cercon/0.5 & $17.66 \pm 3.3^{1 \mathrm{Ka}}$ & $28.80 \pm 2.3^{2 \mathrm{Xe}}$ \\
\hline Cercon/1.0 & $16.73 \pm 2.8^{1 \mathrm{KLb}^{*}}$ & $27.11 \pm 2.5^{2 X^{*} *}$ \\
\hline Cercon/1.5 & $14.47 \pm 2.4^{1 \mathrm{LMc} *}$ & $24.70 \pm 2.7^{2 \mathrm{Yj}^{*}}$ \\
\hline Cercon/2.0 & $13.15 \pm 3.1^{1 \mathrm{Md}^{*}}$ & $21.82 \pm 2.4^{2 \mathrm{Zm} *}$ \\
\hline
\end{tabular}

Different numbers in the same row indicate significant difference $(P<0.05)$ between 2 resin cements. Different uppercase letters in the same column indicate significant difference $(P<0.05)$ among ceramic thicknesses in each ceramic type.

Different lowercase letters in the same column indicate significant difference $(P<0.05)$ among ceramic types in each ceramic thickness.

* Indicates significant difference $(P<0.05)$ from control group.

of the control group. The surface hardness of NX3, polymerized under IPS e.max Press HT $2.0 \mathrm{~mm}$, LT and MO at a thickness level of $1.5,2.0 \mathrm{~mm}$, and $\mathrm{HO}$ and Cercon at a thickness level of $1.0,1.5$, and $2.0 \mathrm{~mm}$ was significantly lower than that of control. Furthermore, the surface hardness of both resin cements when polymerized under each ceramic type showed statistically significant differences between thicknesses. Resin cement polymerized under $2.0 \mathrm{~mm}$ ceramic discs tended to have the lowest surface hardness value in each ceramic.

When comparing the surface hardness value of two resin cements polymerized under different ceramic types with the same thickness, the surface hardness of Variolink Veneer in all thicknesses of ceramic showed no statistically significant differences among all ceramic types. The resin cement polymerized under IPS e.max Press HT and Cercon tended to have the highest and lowest surface hardness, respectively, through each thickness range. For NX3, the surface hardness of cement polymerized under $0.5 \mathrm{~mm}$ ceramic discs showed no statistically significant differences among all ceramic types. When NX3 was polymerized under 1.0, 1.5, and $2.0 \mathrm{~mm}$ ceramic discs, the surface hardness of resin cement under IPS e.max Press HT was significantly higher than Cercon. However, when NX3 polymerized under $2.0 \mathrm{~mm}$ ceramic discs, the surface hardness of resin cement under both IPS e.max Press HT and MO was higher than Cercon.

\section{Discussion}

The use of resin cement for luting restorations, especially all ceramic restorations, is quite common. Adequate polymerization of resin cement will result in high-bond strength between tooth and restoration. Light intensity is one of the most important factors that affects polymerization of lightcured resin cements. Recent studies have shown a positive correlation between light intensity and the degree of conversion of restorative materials [21-23]. Rueggeberg et al. [12] suggested that the adequate intensity for a light-curing unit is $400 \mathrm{~mW} / \mathrm{cm}^{2}$ in order to initiate polymerization of resinbased material, but is unsuitable when the light-curing unit irradiated light with intensity under $233 \mathrm{~mW} / \mathrm{cm}^{2}$. The ISO [24] also suggested a minimum intensity of $300 \mathrm{~mW} / \mathrm{cm}^{2}$ in the $400-515 \mathrm{~nm}$ wavelength bandwidth. The LED lightcuring unit used in this study provided light intensity up to $1,450 \mathrm{~mW} / \mathrm{cm}^{2}$, as measured by a radiometer, and induced a high degree of polymerization and surface hardness of resin cements. However, when irradiating through ceramic discs, 
the light intensity decreased as a function of the type and thickness of the ceramics [25-27].

The dental ceramics used in this study were IPS e.max Press and Cercon, which are indicated for veneer and crown fabrication. The IPS e.max Press, a lithium disilicate glass ceramic, has 4 types corresponding to opacity: high translucency (HT), low translucency (LT), medium opacity (MO), and high opacity $(\mathrm{HO})$. Cercon is one of the CAD/CAM zirconia ceramics possessing high strength and opacity [1]. In this study, when irradiating light through Cercon and IPS e.max Press, the light intensity through Cercon was lower compared with IPS e.max Press, which has lower opacity.

Surface hardness is one of the most effective methods to evaluate the polymerization of resin cement. Many studies have shown that Knoop hardness has a positive correlation with the degree of conversion of resin cement $[15,17]$. Furthermore, Knoop hardness has also been reported to be related to the light intensity of the light-curing unit $[20,27]$. Resin cement which received higher light intensity had better polymerization and higher Knoop hardness than that receiving lower light intensity. In this study, surface hardness was measured at the bottom surface of specimens, opposite to the light activation surface, to show the degree of polymerization of the whole material. A study by Aguiar et al. [28] has shown the surface hardness of the top surface was the highest. It decreased significantly moving from the top toward the bottom of the specimen due to greater distance from the light guide. Moreover, resin cement can disperse light from the lightcuring unit as resin matrix and filler particle scatter the light and thus reduce light intensity when passing through resin cement $[13,29]$. Consequently, the surface hardness of the top surface does not indicate the hardness of other portions or degree of conversion of materials.

Resin cement polymerized under ceramic discs received lower light intensity as the thicknesses and opacity of ceramic increased. The decrease of surface hardness was statistically significant as a function of change in those parameters [30]. This study found that direct activation resin cement had higher surface hardness than resin cement which was activated through ceramic, especially Variolink Veneer polymerized under Cercon 1.0, 1.5, and $2.0 \mathrm{~mm}$. While both resin cements were polymerized under all ceramic types of 0.5 and $1.0 \mathrm{~mm}$ thickness, the surface hardness did not vary from the surface hardness of the control group except NX3 polymerized under IPS e.max Press HO $1.0 \mathrm{~mm}$ and Variolink Veneer polymerized under Cercon $1.0 \mathrm{~mm}$. For ceramic thicknesses of 1.5 and $2.0 \mathrm{~mm}$, the surface hardness was different from that of the control group where resin cement was polymerized under ceramic with higher opacity, such as IPS e.max Press HO and Cercon. This may imply that the thickness of ceramic has less effect on high-translucency ceramics than low-translucency or high-opacity ceramics. The surface hardness of resin cements polymerized under high-opacity ceramic was lower than those polymerized under lower-opacity ceramic. The surface hardness of both resin cements polymerized under Cercon zirconia ceramic showed the lowest hardness, while resin cement polymerized under IPS e.max Press HT, a glass ceramic, tended to have the highest hardness of all thicknesses. These results are in line with the finding of Borges et al. [31] who reported that resin cement polymerized under alumina and zirconia ceramic had lower hardness than cement polymerized under glass ceramic. Furthermore, the study found that NX3 had higher hardness than Variolink Veneer in all groups. These results are likely due to different compositions of resin cement such as types, quantities, and size of filler, which affect polymerization of materials $[32,33]$.

\section{References}

[1] H. J. Conrad, W. J. Seong, and I. J. Pesun, "Current ceramic materials and systems with clinical recommendations: a systematic review," Journal of Prosthetic Dentistry, vol. 98, no. 5, pp. 389-404, 2007.

[2] K. A. Malament and S. S. Socransky, "Survival of Dicor glassceramic dental restorations over 16 years-part III: effect of luting agent and tooth or tooth-substitute core structure," Journal of Prosthetic Dentistry, vol. 86, no. 5, pp. 511-519, 2001.

[3] C. Shen, "Dental cements," in Phillips' Science of Dental Materials, K. J. Anusavice, Ed., Saunders, Philadelphia, Pa, USA, 11th edition, 2003.

[4] W. F. Caughman, G. B. Caughman, R. A. Shiflett, F. Rueggeberg, and G. S. Schuster, "Correlation of cytotoxcity, filler loading and curing time of dental composites," Biomaterials, vol. 12, no. 8, pp. 737-740, 1991.

[5] J. A. Pires, E. Cvitko, G. E. Denehy, and E. J. Swift Jr., "Effects of curing tip distance on light intensity and composite resin microhardness," Quintessence International, vol. 24, no. 7, pp. 517-521, 1993.

[6] R. Janda, J. F. Roulet, M. Kaminsky, G. Steffin, and M. Latta, "Color stability of resin matrix restorative materials as a function of the method of light activation," European Journal of Oral Sciences, vol. 112, no. 3, pp. 280-285, 2004.

[7] M. Goldberg, "In vitro and in vivo studies on the toxicity of dental resin components: a review," Clinical Oral Investigations, vol. 12, no. 1, pp. 1-8, 2008.

[8] I. E. Ruyter and H. Oysaed, "Conversion in different depths of ultraviolet and visible light activated composite materials," Acta Odontologica Scandinavica, vol. 40, no. 3, pp. 179-192, 1982.

[9] R. Nomoto, "Effect of light wavelength on polymerization of light-cured resins," Dental Materials Journal, vol. 16, no. 1, pp. 60-73, 1997.

[10] M. Taira, H. Urabe, T. Hirose, K. Wakasa, and M. Yamaki, "Analysis of photo-initiators in visible-light-cured dental composite resins," Journal of Dental Research, vol. 67, no. 1, pp. 24-28, 1988.

[11] D. Dietschi, N. Marret, and I. Krejci, "Comparative efficiency of plasma and halogen light sources on composite microhardness in different curing conditions," Dental Materials, vol. 19, no. 6, pp. 493-500, 2003.

[12] F. A. Rueggeberg, W. F. Caughman, and J. W. Curtis, "Effect of light intensity and exposure duration on cure of resin composite," Operative Dentistry, vol. 19, no. 1, pp. 26-32, 1994.

[13] A. U. Yap, "Effectiveness of polymerization in composite restoratives claiming bulk placement: impact of cavity depth and exposure time," Operative Dentistry, vol. 25, no. 2, pp. 113$120,2000$.

[14] R. W. Mills, A. Uhl, G. B. Blackwell, and K. D. Jandt, "High power light emitting diode (LED) arrays versus halogen light 
polymerization of oral biomaterials. Barcol hardness, compressive strength and radiometric properties," Biomaterials, vol. 23, no. 14, pp. 2955-2963, 2002.

[15] J. L. Ferracane, "Correlation between hardness and degree of conversion during the setting reaction of unfilled dental restorative resins," Dental Materials, vol. 1, no. 1, pp. 11-14, 1985.

[16] J. L. Ferracane, P. Aday, H. Matsumoto, and V. A. Marker, "Relationship between shade and depth of cure for lightactivated dental composite resins," Dental Materials, vol. 2, no. 2, pp. 80-84, 1986.

[17] F. A. Rueggeberg and R. G. Craig, "Correlation of parameters used to estimate monomer conversion in a light-cured composite," Journal of Dental Research, vol. 67, no. 6, pp. 932-937, 1988.

[18] R. R. Moraes, W. C. Brandt, L. Z. Naves, L. Correr-Sobrinho, and E. Piva, "Light- and time-dependent polymerization of dual-cured resin luting agent beneath ceramic," Acta Odontologica Scandinavica, vol. 66, no. 5, pp. 257-261, 2008.

[19] F. H. Rasetto, C. F. Driscoll, and J. A. Von Fraunhofer, "Effect of light source and time on the polymerization of resin cement through ceramic veneers," Journal of Prosthodontics, vol. 10, no. 3, pp. 133-139, 2001.

[20] G. C. Santos Jr., O. El-Mowafy, J. H. Rubo, and M. J. M. C. Santos, "Hardening of dual-cure resin cements and a resin composite restorative cured with QTH and LED curing units," Journal of the Canadian Dental Association, vol. 70, no. 5, pp. 323-328, 2004.

[21] J. A. Yearn, "Factors affecting cure of visible light activated composites," International Dental Journal, vol. 35, no. 3, pp. 218-225, 1985.

[22] J. F. McCabe and T. E. Carrick, "Output from visible-light activation units and depth of cure of light-activated composites," Journal of Dental Research, vol. 68, no. 11, pp. 1534-1539, 1989.

[23] C. S. Fowler, M. L. Swartz, and B. K. Moore, "Efficacy testing of visible-light-curing units," Operative Dentistry, vol. 19, no. 2, pp. 47-52, 1994.

[24] "International Organization for Standardization ISO/TS 10650:1999. Dental equipment-powered polymerization activators," International Organization for Standardization, Geneva, Switzerland, 1999.

[25] O. M. el-Mowafy, M. H. Rubo, and W. A. el-Badrawy, "Hardening of new resin cements cured through a ceramic inlay," Operative Dentistry, vol. 24, no. 1, pp. 38-44, 1999.

[26] H. Jung, K. H. Friedl, K. A. Hiller, H. Furch, S. Bernhart, and G. Schmalz, "Polymerization efficiency of different photocuring units through ceramic discs," Operative Dentistry, vol. 31, no. 1, pp. 68-77, 2006.

[27] F. H. Rasetto, C. F. Driscoll, V. Prestipino, R. Masri, and J. A. Von Fraunhofer, "Light transmission through all-ceramic dental materials: a pilot study," Journal of Prosthetic Dentistry, vol. 91, no. 5, pp. 441-446, 2004.

[28] F. H. B. Aguiar, A. Braceiro, D. A. N. L. Lima, G. M. B. Ambrosano, and J. R. Lovadino, "Effect of light curing modes and light curing time on the microhardness of a hybrid composite resin," Journal of Contemporary Dental Practice, vol. 8, no. 6, pp. 1-8, 2007.

[29] F. Rueggeberg, "Contemporary issues in photocuring," Compendium of Continuing Education in Dentistry, no. 25, supplement, pp. S4-S15, 1999.

[30] S. Uctasli, U. Hasanreisoglu, and H. J. Wilson, "The attenuation of radiation by porcelain and its effect on polymerization of resin cements," Journal of Oral Rehabilitation, vol. 21, no. 5, pp. 565-575, 1994.

[31] G. A. Borges, P. Agarwal, B. A. S. Miranzi, J. A. Platt, T. A. Valentino, and P. H. Santos, "Influence of different ceramics on resin cement Knoop Hardness Number," Operative Dentistry, vol. 33, no. 6, pp. 622-628, 2008.

[32] N. Hofmann, G. Papsthart, B. Hugo, and B. Klaiber, "Comparison of photo-activation versus chemical or dual-curing of resin-based luting cements regarding flexural strength, modulus and surface hardness," Journal of Oral Rehabilitation, vol. 28, no. 11, pp. 1022-1028, 2001.

[33] R. B. T. Price, C. A. Felix, and P. Andreou, "Effects of resin composite composition and irradiation distance on the performance of curing lights," Biomaterials, vol. 25, no. 18, pp. 4465-4477, 2004. 


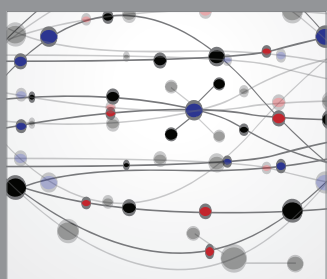

The Scientific World Journal
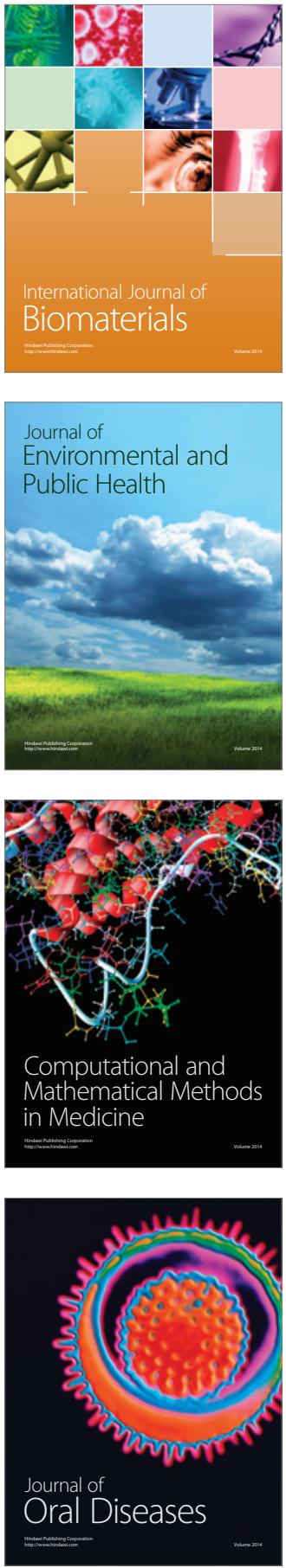
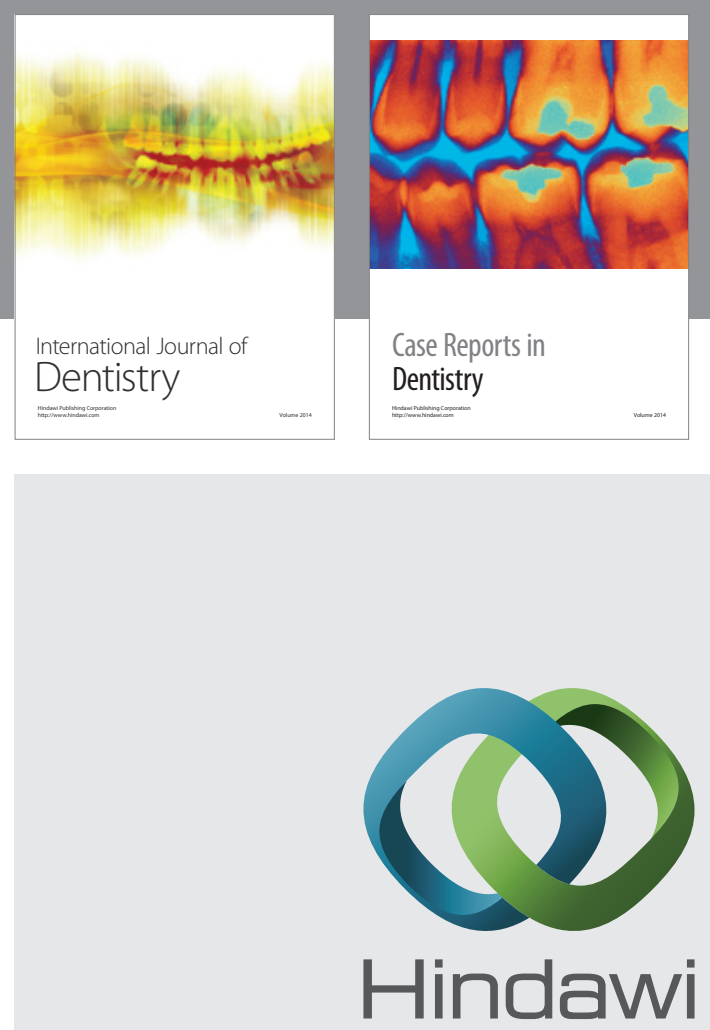

Submit your manuscripts at

http://www.hindawi.com
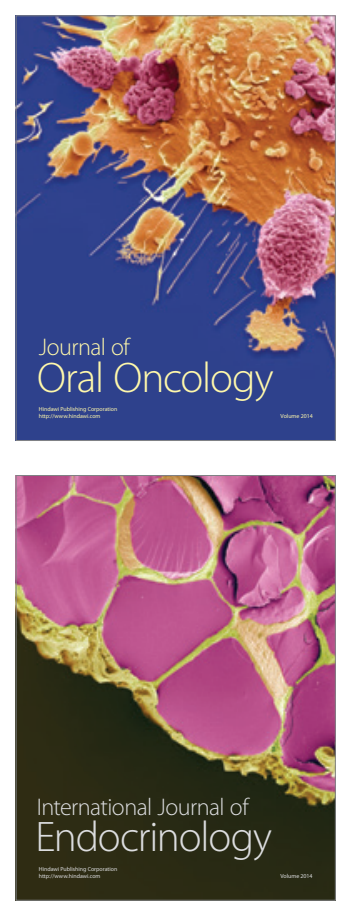
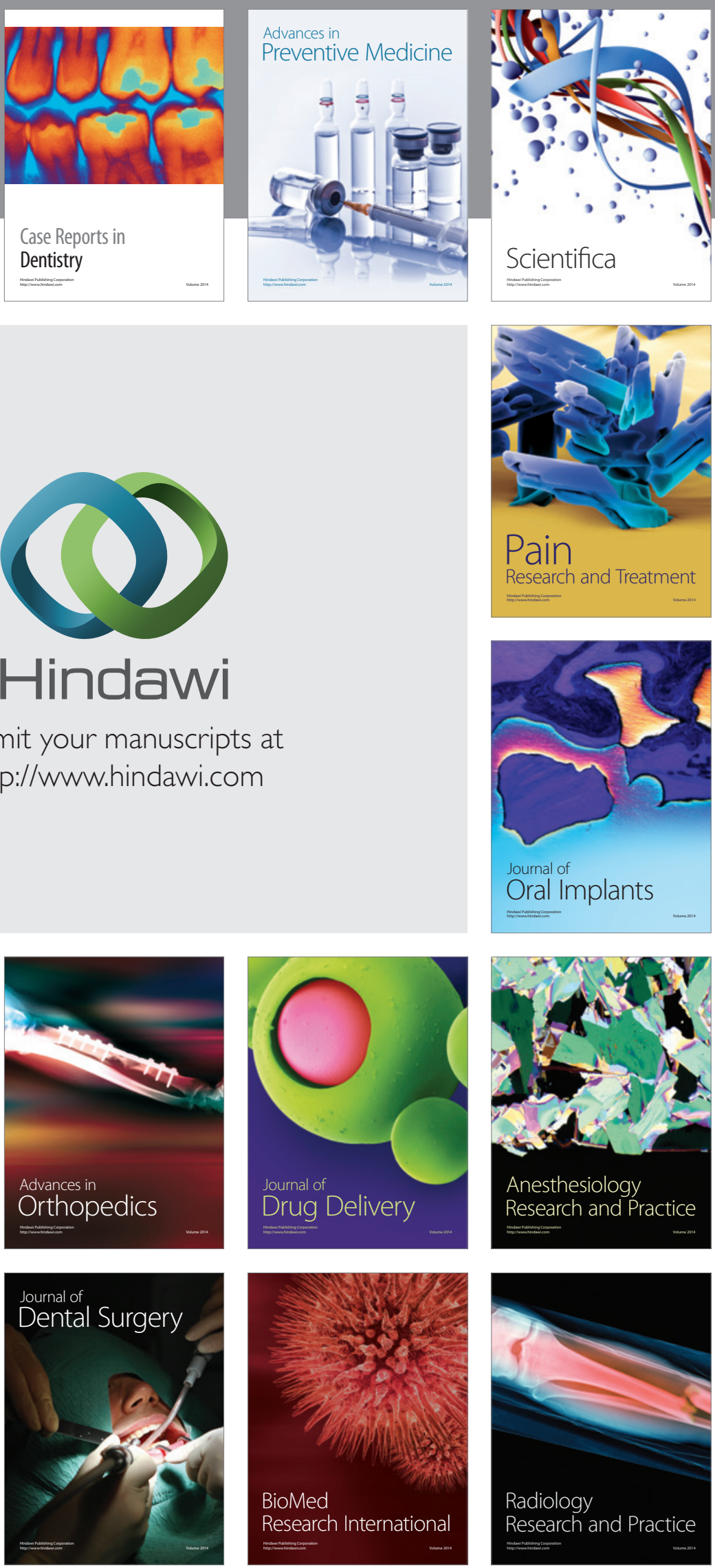